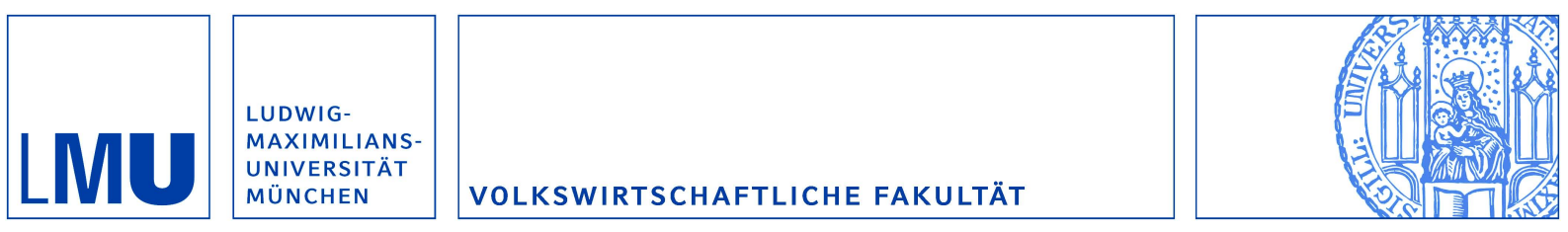

Mastromarco, Camilla and Runkel, Marco:

Rule Changes and Competitive Balance in Formula One Motor Racing

Munich Discussion Paper No. 2004-16

Department of Economics

University of Munich

Volkswirtschaftliche Fakultät

Ludwig-Maximilians-Universitäł München

Online at https://doi.org/10.5282/ubm/epub.386 


\title{
Rule Changes and Competitive Balance in Formula One Motor Racing ${ }^{\mathrm{a}}$
}

\author{
Camilla Mastromarco ${ }^{\mathrm{b}}$ \\ Department of Economics and Quantitative Methods, University of Leece \\ Marco Runkel ${ }^{\mathrm{c}}$ \\ Department of Economics, University of Munich
}

June 28, 2004

\begin{abstract}
This paper provides an economic explanation of the frequent rule changes in the Formula One (F1) motor racing series. In a two-stage model, the FIA (as the organizer of the F1) first decides whether to change the rules or not, and then the racing teams compete in a contest. It turns out that a rule change reduces the teams' performances, but also improves competitive balance between the teams. The rule change is implemented, if the FIA's revenue gain from the latter effect overcompensates the FIA's revenue loss from the former effect. We provide empirical evidence from F1 seasons in the period 1950-2003 which supports the main implications of the model.
\end{abstract}

JEL classification: $\quad$ D43, L83

key words: $\quad$ Formula One, Competitive Balance, Contest

${ }^{a}$ The present study was carried out while Camilla Mastromarco was Marie Curie Research Fellow at the Department of Economics, University of Munich. We thank Ulrich Woitek for several comments which considerably improved the paper. We also appreciate the helpful suggestions of seminar participants at the University of Leece and at the University of Munich. All errors remain our own.

${ }^{\mathrm{b}}$ Camilla Mastromarco, Department of Economics and Quantitative Methods, University of Leece, Ecotekne, via per Monteroni, 73100 Leece, Italy, email: c.mastromarco@economica.unile.it

${ }^{c}$ Marco Runkel, Department of Economics, University of Munich, Ludwigstr. 28/Vgb./III, 80539 Munich, Germany, email: marco.runkel@lrz.uni-muenchen.de (corresponding author) 


\section{Introduction}

The Formula One (F1) motor racing series has become big business. It started with the 1950 season, in which 7 races were followed by spectators at the circuits only. Today, a F1 season consists of 17 races and its television audience runs to billions. According to FIA (1999), for instance, the races of the 1999 season (including training and qualifying) attracted over 57 billion television viewers in 206 different countries. Most league sports like soccer or football have a smaller audience since they are usually subject to national interest only. The Soccer World Championship and the Olympic Games reach similar television rates. But they take place every four years only. The F1 series is therefore one of the world's most followed sport events. By selling broadcasting rights and attracting sponsors, the organizer of the F1 series, the Fédération Internationale de l'Automobile (FIA), transforms this fan interest into a huge revenue. It is estimated that the FIA's average annual TV revenue (without other revenue from e.g. merchandising and sponsoring) amounts to 510 million US\$. ${ }^{1}$

A remarkable feature of the F1 series is the tendency to frequent rule changes. In the past 13 years there were on average 8 changes per season. ${ }^{2}$ It is apparent that these rule changes often impede the performance of the racing teams. Examples are all regulations which restrict the technical capability of the cars, e.g. the definition of a maximum bodywork height/width in 1969 and a minimum cockpit dimensions in 1972, the ban of electronic control in 1994 and the reduction of engine capacity in 1995. The FIA as the rule setting organization often justifies this kind of regulation by the safety of drivers and, indeed, the F1 races have become considerably safer. Today, only one in every 300 accidents is serious or fatal, compared with one in every ten accidents in the 1950s and 1960s. But there were other rule changes which can hardly be justified by safety arguments. In the 2003 season, for example, the number of qualifying rounds per driver was reduced to one and the teams were no longer allowed to change settings of the cars between the qualifying session and the race. Hence, the teams could not improve the setup of the car by e.g. changing tyres or quantity of fuel. Such rule

\footnotetext{
${ }^{1}$ Day, J., 'Formula One Teams to Fight Pay-TV Plans', Media Guardian, February 22, 2002.

${ }^{2}$ Detailed information on rule changes and the information on accidents which we provide below can be found on the website http://www.f1technical.net.
} 
changes usually reduce the performance of the teams, but have less to do with the safety of drivers.

The major aim of this paper is to provide an economic explanation of such rule changes. We develop a two-stage model of a F1 season. At the first stage, the FIA decides whether to change the rules or not. In doing so, it maximizes its broadcasting revenue which is positively correlated with the fan interest. The fan interest, in turn, is increasing in the performance of the racing teams and in competitive balance between the teams. The FIA's decision influences the outcome at the second stage where racing teams with different abilities compete for a trophy money which the FIA finances out of its broadcasting revenue. The team competition is modelled as a contest first considered in the rent-seeking literature by Tullock (1980). A rule change is represented either by a uniform reduction in the teams' abilities or by a reduction in the discriminatory power of the contest. A decrease in the discriminatory power means that the outcome of the team contest is determined to a larger part by chance. It turns out that rule changes reduce the teams' performances, but also improve competitive balance. Consequently, the FIA decides to implement the rule change, if its revenue gain from the latter effect outweighs its revenue loss from the former effect.

Using a dataset on the F1 seasons 1950-2003, we empirically test this theoretical model by estimating its two main implications. First, the theoretical model predicts that rule changes at the beginning of a season are the more likely and the more comprehensive, the smaller competitive balance has been in the previous season. We test this implication by using a Poisson model and show that a unit increase in the standard deviation of points scored by the teams in a F1 season significantly raises the expected number of rule changes in the next season by about $3 \%$. Second, we use an OLS regression to test the implication that rule changes at the beginning of a season improve competitive balance during the season. This will be done for both kinds of rule changes which the FIA distinguishes, namely the so-called 'safety regulations' and 'other regulations'. We find a significantly positive impact of the rule changes on competitive balance. A $10 \%$ increase in the number of safety regulations reduces the standard deviation of points scored by the racing teams by $2.8 \%$, while a $10 \%$ increase in the number of other regulations reduces this standard deviation even by $5.6 \%$. 
In recent years, there is a rapidly growing literature on sports economics (for excellent surveys see Fort and Quirk, 1995, and Szymanski, 2003), but we are unaware of any study focusing on (F1) motor racing. This literature mainly analyses the impact of specific measures like e.g. revenue sharing on competitive balance in league sports. Studies inquiring into the optimal regulation of the discriminatory power or the contestants' abilities are not available. Also the growing literature on optimal contest design (e.g. Dasgupta and Nti, 1998, Amegashi, 1999) does not yet provide such studies. An exception is Michaels (1988) who considers the optimal choice of the discriminatory power in a symmetric contest. Since all players are identical, however, his contest is always balanced and there is no scope for improvements in competitive balance which is the driving force of our results.

The paper is organized as follows. In Section 2 we briefly describe the organization and the basic rules of the F1 series. Section 3 develops and analyses the theoretical model. The predictions of this model are tested in Section 4 and Section 5 concludes.

\section{Organization and Basic Rules of the F1}

The controlling organization of the F1 motor racing series is the Fédération Internationale de l'Automobile (FIA) with the headquarters in Paris. ${ }^{3}$ One of the main tasks of the FIA is to set the rules of the F1 series. It usually decides before a new season whether and, if so, which rules of the previous season should be changed. Another important task of the FIA is the marketing of the broadcasting rights of the F1 series. Until 1996, the FIA itself sold these rights to broadcasting stations. In 1996, the FIA handed these rights for a period of 14 years to private companies owned by Bernie Ecclestone. But Ecclestone is one of the vice presidents of the FIA, and in this position he is jointly responsible for the rule setting. Hence, also after 1996 the rule setting institution (the FIA) is in some sense responsible for selling the broadcasting rights.

There are several teams competing in the F1 series. A team is allowed to participate with two cars. It employs mechanics who set up the cars and the other technical equipment, and drivers who are still important although the success of a team is by

\footnotetext{
${ }^{3}$ All the following information on the F1 series can be found at the website www.fia.com.
} 
now largely determined by the technical quality of the cars. The main revenue source of the teams is sponsoring. The leading teams usually experience comprehensive support from companies of the automobile industry (Ferrari, BMW, Renault, etc.). Payments received from the FIA represent another important revenue source for the racing teams. The FIA shares a part of its broadcasting revenue with the racing teams according to their rankings at the end of the season. This revenue source covers about $20 \%$ of the teams' total costs.

The ranking of the teams is determined as follows. A F1 season consists of 17 events. An event usually lasts for three days. During the first two days the drivers have free practice and qualifying sessions. The race itself takes place on the third day. The drivers' starting positions in the race are determined by their performance in the qualifying sessions. The driver who registers the fastest qualifying time starts from the pole position followed by the driver with the second qualifying time and so on. The drivers get points according to their rankings at the end of the race. For instance, in the season 2003 the first eight ranks of a race get points which go down from 10 to 1. At the end of a season, the FIA awards two titles. In the drivers' championship, drivers accumulate the points scored in the 17 races of the season and the driver with the most points wins the title. In the constructors' championship, each team adds together the points scored by its drivers and the title is awarded to the team with the largest number of points.

\section{Theoretical Model}

Based on the information given in the previous section, we view a F1 season as a twostage process. At the first stage, the FIA as the organizer of the F1 series decides whether to change the rules of the previous season or not. At the second stage, the racing teams take the rules as given and compete in a contest in hope of obtaining (a part of the) broadcasting revenue collected by the FIA. This two-stage process is solved recursively in order to obtain a subgame-perfect solution. In doing so, we assume that the FIA and the teams are profit maximizer. An argument in favor of profit-maximizing behavior of the racing teams is that they are often under the control of profit-maximizing companies of the automobile industry. The transfer of the 
broadcasting rights to profit-maximizing companies may be seen as an indication that profit considerations play a role in the rule setting of the FIA.

\subsection{Stage 2: Team Competition}

The team competition is modelled as a contest. To make our point, it suffices to focus on a contest with two teams. We explicitly model the constructors' championship only and ignore the drivers' championship. A team is therefore treated as a single player. Effort of team $i=1,2$ is denoted by $e_{i}$. It reflects the salary of the drivers and the cost of developing and producing the cars (e.g. material cost and the salary of the mechanics). Team $i$ 's effective amount of effort is represented by $\alpha_{i} e_{i}$. The parameter $\alpha_{i}$ may be interpreted as team $i$ 's ability of transforming effort into effective units. We assume $\alpha_{1}>\alpha_{2}$, i.e. team 1 is more able than team 2. Such a difference in abilities may have several reasons. For example, a team might have better engineers which develop a more powerful car than the engineers of the other team. Another reason, which we will refer to below, is the different success in previous seasons. It is often the case that a team is the more experienced and thus the more able in the present season, the more successful it has been in the past. Such a 'learning-by-winning' effect is in particular relevant for the F1 series since the ability to realize a good performance of the cars and the drivers depends to a high degree on trial and error.

The performance of team $i$ is denoted by $h_{i}$. It is an increasing function of team $i$ 's effective effort according to the relation $h_{i}=\left(\alpha_{i} e_{i}\right)^{r}$ with $r>0$. Performance $h_{i}$ can best be viewed as an aggregate of several indicators which describe the characteristics of team $i$ 's cars and drivers. Examples for such indicators are the maximum speed, the horsepower or the air drag coefficient of team $i$ 's car, the physical and mental strength of team $i$ 's drivers, the minimum time a driver of team $i$ needs for the qualifying round on a 'representative' circuit or the inverse of the failure rate of team $i$ 's cars during the races of the $\mathrm{F} 1$ season. More loosely speaking, $h_{i}$ reflects the ability of team $i$ to optimize the interaction between the drivers' and the cars' performance such that they perform well in a typical race of the F1 series.

The teams expend resources in hope of winning (a part of) the trophy money $v>$ 0 . This trophy money is financed by the FIA out of its revenue from selling the 
broadcasting rights. It is divided among the teams according to their success during the season. To model such success-orientated payments in a simple way, we assume that each team obtains a share of $v$ which reflects the team's share at total points. For example, if a team achieves $20 \%$ of total points, it also gets $20 \%$ of the trophy money $v$. Furthermore, we assume that team $i$ 's share at total points, $p_{i}$, equals the share of its performance at total performance of both teams, i.e.

$$
p_{i}=\frac{h_{i}}{h_{i}+h_{j}}=\frac{\left(\alpha_{i} e_{i}\right)^{r}}{\left(\alpha_{i} e_{i}\right)^{r}+\left(\alpha_{j} e_{j}\right)^{r}}, \quad i, j=1,2, \quad i \neq j .
$$

The larger team $i$ 's performance relatively to team $j$ 's performance, the larger is team $i$ 's share at total points. From an ex ante point of view, $p_{i}$ can be interpreted as team $i$ 's winning probability and $p_{i} v$ is expected trophy money of team $i$.

According to equation (1), the parameter $r$ may be interpreted as discriminatory power of the contest. It measures to what extent the contest outcome is determined by chance and to what extent by the teams' effort levels. In the extreme case of $r \rightarrow \infty$, the contest is perfectly discriminating. Effort is then the only determinant of the contest outcome and the team with the higher effective effort level wins all points. For instance, if $\alpha_{i} e_{i}>\alpha_{j} e_{j}$, then $r \rightarrow \infty$ implies $p_{i}=1$ and $p_{j}=0$. In contrast, effort does not influence the contest outcome at all if $r \rightarrow 0$. The winning probabilities of the teams are then $p_{i}=p_{j}=1 / 2$ and the contest outcome is determined solely by chance. To put it another way, the smaller the discriminatory power $r$, the less sensitive is the contest outcome to variations in effort levels and the larger is the influence of chance.

Expected profit of team $i$ equals the team's expected trophy money less the cost of effort. ${ }^{4}$ If the latter is assumed to be identical to the effort level, then team $i$ 's expected profit may be written as

$$
\Pi^{i}\left(e_{i}, e_{j}\right)=p_{i} v-e_{i}=\frac{\left(\alpha_{i} e_{i}\right)^{r}}{\left(\alpha_{i} e_{i}\right)^{r}+\left(\alpha_{j} e_{j}\right)^{r}} v-e_{i}, \quad i, j=1,2, \quad i \neq j .
$$

Team $i$ chooses effort such that its profit (2) is maximized. In doing so, it takes as given the effort of team $j$ and the rules set by the FIA at stage 1 of the F1 season.

\footnotetext{
${ }^{4}$ For simplicity, we ignore other important revenue sources of the teams like sponsoring and advertising. It is straightforward to include these sources in our model. But this would merely complicate the analysis without adding further insights.
} 
As will be argued in more detail below, the rules of the contest determine (or at least influence) the parameters $v, r, \alpha_{1}$ and $\alpha_{2}$.

In solving the team contest, we look for a pure-strategy Nash-equilibrium. The equilibrium is determined by the first- and second-order conditions of the teams' profit maximization. These conditions read

$$
\begin{gathered}
\Pi_{e_{i}}^{i}\left(e_{i}, e_{j}\right)=\frac{r \alpha_{i}\left(\alpha_{i} e_{i}\right)^{r-1}\left(\alpha_{j} e_{j}\right)^{r}}{\left[\left(\alpha_{i} e_{i}\right)^{r}+\left(\alpha_{j} e_{j}\right)^{r}\right]^{2}} v-1=0, \\
\prod_{e_{i} e_{i}}^{i}\left(e_{i}, e_{j}\right)=\frac{r \alpha_{i}^{2}\left(\alpha_{i} e_{i}\right)^{r-2}\left(\alpha_{j} e_{j}\right)^{r}\left[(r-1)\left(\alpha_{j} e_{j}\right)^{r}-(r+1)\left(\alpha_{i} e_{i}\right)^{r}\right]}{\left[\left(\alpha_{i} e_{i}\right)^{r}+\left(\alpha_{j} e_{j}\right)^{r}\right]^{3}} v<0,
\end{gathered}
$$

for $i, j=1,2$ and $i \neq j$. The first-order condition (3) immediately implies that in equilibrium both teams choose the same effort, i.e. $e_{1}=e_{2}=: e^{*}$. The closed-form solution for equilibrium effort can be computed from (3) as

$$
e^{*}=\frac{r \alpha_{1}^{r} \alpha_{2}^{r} v}{\left(\alpha_{1}^{r}+\alpha_{1}^{r}\right)^{2}}
$$

Since team 1 is more able than team 2, it realizes a higher performance and a higher chance of winning. Inserting $e^{*}$ into (1) gives the equilibrium winning probabilities

$$
p_{1}^{*}=\frac{\alpha_{1}^{r}}{\alpha_{1}^{r}+\alpha_{2}^{r}}>\frac{\alpha_{2}^{r}}{\alpha_{1}^{r}+\alpha_{2}^{r}}=p_{2}^{*}
$$

Using (5) and (6) in (2) yields equilibrium profits

$$
\Pi^{1 *}=\frac{\alpha_{1}^{r}\left[\alpha_{1}^{r}+(1-r) \alpha_{2}^{r}\right]}{\left(\alpha_{1}^{r}+\alpha_{2}^{r}\right)^{2}} v>\frac{\alpha_{2}^{r}\left[\alpha_{2}^{r}+(1-r) \alpha_{1}^{r}\right]}{\left(\alpha_{1}^{r}+\alpha_{2}^{r}\right)^{2}} v=\Pi^{2 *} .
$$

Hence, equilibrium profit of team 1 is larger than that of team 2 . For $e^{*}$ to be a pure-strategy Nash-equilibrium, profits of both teams have to be non-negative at $e^{*}$. Equation (7) implies that $\Pi^{1 *}>\Pi^{2 *} \geq 0$ if and only if

$$
r \leq 1+\alpha_{2}^{r} / \alpha_{1}^{r}<2
$$

We suppose the parameters $r, \alpha_{1}$ and $\alpha_{2}$ satisfy this condition throughout. Note that the second-order condition (4) is then satisfied as well, implying both teams attain a profit maximum at $e^{*}$. To sum up, if condition (8) is satisfied, then $e^{*}$ from (5) is the unique Nash-equilibrium of the team contest. 


\subsection{Stage 1: Contest Regulation}

Having solved the team contest, we now turn to the rule setting of the FIA at the first stage of the F1 season. The larger the fan interest in the F1 races, the larger is the profit a broadcasting station can realize with the TV coverage of the races and the larger is the willingness-to-pay of the station for the broadcasting rights of the F1 series. The revenue of the FIA from selling broadcasting rights is therefore positively correlated with the fan interest in the F1 series. The fan interest, in turn, is increasing in two variables. First, fans are interested in races with high performances of the teams. For example, if the maximum speed of the cars is high, the number of viewers will usually be larger than in case where the cars are quite gammy. Second, fans like close competition. The more uncertain the outcome of the F1 season, the more exciting is the season and the larger is the number of people who are willing to follow the races. To put it the other way round, if it is almost clear from the outset which team will win the championship, then the F1 season is rather uninteresting for the fans.

To model these properties, we assume that the FIA's revenue from selling the broadcasting rights is a function $R\left(a^{*}, b^{*}\right)$ where

$$
a^{*}=h_{1}^{*}+h_{2}^{*}=\frac{\left(r v \alpha_{1}^{r} \alpha_{2}^{r}\right)^{r}}{\left(\alpha_{1}^{r}+\alpha_{2}^{r}\right)^{2 r-1}}
$$

is total performance of both teams in the contest equilibrium and where

$$
b^{*}=p_{1}^{*}-p_{2}^{*}=\frac{\alpha_{1}^{r}-\alpha_{2}^{r}}{\alpha_{1}^{r}+\alpha_{2}^{r}}
$$

is the equilibrium difference in winning probabilities of the teams. Note that $b^{*}>0$ due to $\alpha_{1}>\alpha_{2}$. The inverse of $b^{*}$ can be viewed as a measure of the closeness of the F1 season or, equivalently, of competitive balance between the teams. The smaller $b^{*}$, the more equal are the winning probabilities of the teams and the closer or the more balanced is the championship. The FIA's revenue function $R$ is supposed to satisfy

$$
\begin{gathered}
R_{a}\left(a^{*}, b^{*}\right)>0, \quad R_{a a}\left(a^{*}, b^{*}\right) \leq 0, \\
R_{b}\left(a^{*}, b^{*}\right) \gtreqless 0 \Leftrightarrow b \lesseqgtr 0, \quad R_{b b}\left(a^{*}, b^{*}\right)<0
\end{gathered}
$$

where subscripts indicate partial derivatives. Due to (11), the FIA's revenue is increasing and non-convex in total performance. Equation (12) states that, for given total 
performance, the FIA's revenue is maximized in the even contest with both teams having the same winning probability. Deviations from the even contest reduce the FIA's revenue at increasing rates. These properties of $R$ reflect the above mentioned impact of total performance and competitive balance on the FIA's revenue.

Profit of the FIA may be written as $R\left(a^{*}, b^{*}\right)-v$. It equals the broadcasting revenue of the FIA less the trophy money divided among the teams. The FIA sets the rules of the F1 in order to maximize its profit. In doing so, it accounts for the impact the rules have on equilibrium performance and competitive balance specified in (9) and (10), respectively. As already mentioned above, the rules of the contest determine the trophy money $v$, the discriminatory power $r$ and the teams' abilities $\alpha_{1}$ and $\alpha_{2}$. To fix our ideas, we suppose throughout that the trophy money $v$ is exogenously given. Profit maximization is then equivalent to maximization of revenue $R\left(a^{*}, b^{*}\right)$. Moreover, we investigate the regulation of the discriminatory power and the regulation of abilities separately. It should be noted, however, that our results qualitatively remain unchanged when the FIA determines $v, r, \alpha_{1}$ and $\alpha_{2}$ simultaneously.

Regulation of the Teams' Abilities. Let us first suppose the discriminatory power $r$ is exogenously given. The rule setting of the FIA may then influence the teams' abilities $\alpha_{1}$ and $\alpha_{2}$ only. A prominent example is the one already mentioned in the introduction. Since the season 2003, the F1 teams are no longer allowed to change the setting of the cars after the qualifying. It is therefore not possible to employ experiences gained during the qualifying session in order to adjust the car to the specific conditions of the circuit. Obviously, this rule change negatively affects the teams' abilities $\alpha_{1}$ and $\alpha_{2}$. To model such a rule change, we assume

$$
\alpha_{i}=\frac{1}{c_{i}+\tau}, \quad i=1,2, \quad \text { with } \quad \tau \geq 0 \quad \text { and } \quad c_{1}<c_{2} .
$$

The parameter $c_{i}$ reflects the inverse ability of team $i$ in the status quo, i.e. without a rule change of the FIA. The variable $\tau$ indicates the rule changes. If the FIA chooses $\tau=0$, then the rules of the previous season remain unchanged. In contrast, $\tau>0$ stands for the case in which the FIA decides to change the rules. This rule change is the more comprehensive, the larger is $\tau$. The specification of the teams' abilities in (13) seems to be very special. It has, however, a straightforward interpretation. If we 
define $x_{i}:=\alpha_{i} e_{i}$, then $e_{i}=\left(c_{i}+\tau\right) x_{i}$. Inserting this into team $i$ 's profit function (2) yields $\Pi^{i}(\cdot)=p_{i} v-\left(c_{i}+\tau\right) x_{i}$. Hence, $c_{i}$ may be interpreted as team $i$ 's unit cost of effort and the rule change parameter $\tau$ works like an increase in unit cost.

The FIA sets $\tau$ such that its revenue is maximized. The associated maximization problem reads

$$
\max _{\tau} R\left(a^{*}, b^{*}\right) \quad \text { s.t. } \quad(9),(10) \text { and }(13) .
$$

We want to know whether the optimal value of $\tau$ is zero (no rule change) or strictly positive (rule change). To solve the maximization problem, the FIA needs to figure out the impact of the rule change parameter on total performance and competitive balance in the contest equilibrium at stage 2. From (9), (10) and (13) we obtain

$$
\begin{aligned}
& \operatorname{sign}\left\{\frac{d a^{*}}{d \tau}\right\}=\operatorname{sign}\left\{r\left(c_{2}-c_{1}\right)-\frac{\left(c_{2}+\tau\right)^{1+r}+\left(c_{1}+\tau\right)^{1+r}}{\left(c_{2}+\tau\right)^{1+r}-\left(c_{1}+\tau\right)^{1+r}}\right\}=-1, \\
& \operatorname{sign}\left\{\frac{d b^{*}}{d \tau}\right\}=\operatorname{sign}\left\{c_{1}-c_{2}\right\}=-1 .
\end{aligned}
$$

The sign in (15) follows from the existence condition (8). It implies that total performance is harmed when rules are changed, i.e. when $\tau$ increases from zero to a strictly positive value. But this is not the only consequence of a rule change. According to (16), competitive balance is improved. The reason is that the relative advantage of team 1 (measured by $\alpha_{1} / \alpha_{2}=\left(c_{2}+\tau\right) /\left(c_{1}+\tau\right)$ ) declines. The changes in total performance and competitive balance have two opposing effects on the FIA's revenue. On the one hand, the reduction in total performance lowers the revenue since viewers prefer teams with high performance. On the other hand, the improvement in competitive balance raises the revenue since viewers like close races. If the second effect overcompensates the first effect, then the FIA has an incentive to change the rules of the previous season.

To formally prove this assertion, we consider the Kuhn-Tucker conditions for the profit maximization problem (14). These conditions read

$$
R_{a}(\cdot) \frac{d a^{*}}{d \tau}+R_{b}(\cdot) \frac{d b^{*}}{d \tau} \leq 0, \quad \tau\left[R_{a}(\cdot) \frac{d a^{*}}{d \tau}+R_{b}(\cdot) \frac{d b^{*}}{d \tau}\right]=0, \quad \tau \geq 0
$$

The term containing $R_{a}$ represents the revenue loss of a rule change due to the decrease in total performance. (11) and (15) imply that this term is negative. The term 
containing $R_{b}$ stands for the revenue gain of a rule change due to the improvement in competitive balance. It is positive according to $b^{*}>0,(12)$ and (16). If the latter effect is negligible, then the solution to the Kuhn-Tucker conditions is $\tau^{*}=0$, i.e. no rule change. In contrast, if the revenue gain from the increase in competitive balance is large enough, then $\tau^{*}>0$ and the FIA changes the rules of the previous season.

For our empirical analysis it is desirable to know how the difference in the teams' abilities influences the optimal choice of the FIA. Unfortunately, our model does not allow a closed-form solution for $\tau$ since the discriminatory power $r$ is not necessarily equal to one. But we can gain some insights if we choose a specific functional form of the revenue function and then simulate the FIA's decision. Consider the linear-quadratic revenue function $R(a, b)=\gamma a-\delta b^{2}$. Setting $v=1000, r=0.4, \gamma=1$ and $\delta=200$, we obtain the simulation result summarized in Table 1 . These results indicate that a

Table 1: Simulation Results for the Regulation of Abilities

\begin{tabular}{|c|c|c|c|c|c|c|c|c|c|}
\hline$c_{1}$ & 9 & 8 & 7 & 6 & 5 & 4 & 3 & 2 & 1 \\
\hline$c_{2}$ & 10 & 10 & 10 & 10 & 10 & 10 & 10 & 10 & 10 \\
\hline$\tau^{*}$ & 0 & 0 & 0 & 3.59 & 7.83 & 12.26 & 16.86 & 21.61 & 26.49 \\
\hline
\end{tabular}

rule change is the more likely and the more comprehensive, the larger the difference in abilities. The intuition is straightforward. For a small difference in abilities, the team contest is relatively balanced even without a rule change. The negative effect of a rule change on total performance would than be more important than the positive effect on competitive balance and the FIA does not have an incentive to alter the rules. In contrast, if team 1's predominance is large enough, competitive balance without a rule change is quite worse. The FIA can then gain more revenue if she tightens the rules such that the teams' abilities decline and the races become closer. Further simulations show that this result is very robust against variations in the parameter values. In fact, we did not succeed in constructing a counterexample.

It should be noted, that a similar result is obtained in Runkel (2003), but the focus there is on the case $r=1$. Our simulations indicate that the result remains true for other values of $r$. This is important since we now turn to the case where the FIA may regulate the discriminatory power such that $r$ need not necessarily be equal to unity. 
Regulation of the Discriminatory Power. Suppose now the abilities of the teams are fixed and the FIA only has the option of regulating the discriminatory power $r$. The FIA frequently used this kind of regulation in the past. The most obvious example has already been mentioned in the introduction. Since the season 2003, the qualifying on the day before the race consists of one round per driver only. The outcome of the qualifying and, thus, the outcome of the race itself is therefore much more influenced by chance. For example, if a driver makes a mistake in his only qualifying round or if the weather conditions in this round are fairly bad, it is no longer possible to try better in another round. This kind of regulation can be modelled by assuming

$$
r=\rho-\sigma \quad \text { with } \quad \sigma \geq 0
$$

$\rho$ reflects the discriminatory power in the status quo without a rule change. The rule change variable is now represented by $\sigma$. If the FIA chooses $\sigma=0$, then the rules remain unchanged. In contrast, the case $\sigma>0$ indicates a rule change which reduces the discriminatory power, i.e. which makes the contest outcome more dependent on chance and less dependent on the teams' effort levels.

The FIA's profit maximization now reads

$$
\max _{\sigma} R\left(a^{*}, b^{*}\right) \quad \text { s.t. } \quad(9),(10) \text { and }(18) .
$$

To solve this problem, the FIA needs to know the impact of the rule change parameter $\sigma$ on the equilibrium of the team contest. The impact of $\sigma$ on equilibrium performance is not unique. From (9) and (18) it can be shown that $d a^{*} / d \sigma$ is negative, if the difference in abilities is sufficiently large. But it may be positive, if the difference in abilities is relatively small. With respect to competitive balance we obtain from (10) and (18)

$$
\operatorname{sign}\left\{\frac{d b^{*}}{d \sigma}\right\}=\operatorname{sign}\left\{\ln \alpha_{2}-\ln \alpha_{1}\right\}<0 .
$$

Hence, competitive balance is improved when the discriminatory power declines through a decrease in $\sigma$. The reason is straightforward. A reduction in the discriminatory power means that the outcome of the team contest is less sensitive to variations in the teams' effort levels. The advantage of team 1 due to its higher ability then becomes less important and competitive balance increases. 
This positive effect of reducing the discriminatory power allows the same argument as in case of ability regulation. Tightening the rules may exert a negative effect on the FIA's revenue, if total performance declines. But there is also a positive effect on the FIA's revenue since the championship becomes closer. If the latter effect overcompensates the former, then the FIA has an incentive to change the rules of the previous season. Formally, this can be seen from the Kuhn-Tucker conditions of problem (20). These conditions are the same as the conditions in (17), except for replacing $\tau$ by $\sigma$. To figure out the impact of the difference in abilities on the FIA's choice, we are again restricted to numerical simulation. Consider the linear-quadratic specification of the revenue function and suppose $v=20$ and $\rho=\gamma=\delta=1$. The simulation results are summarized in Table 2. We obtain similar results as in case where the FIA regulates

Table 2: Simulation Results for the Regulation of Discriminatory Power

\begin{tabular}{|c|c|c|c|c|c|c|c|c|c|}
\hline$\alpha_{1}$ & 10 & 30 & 50 & 70 & 90 & 110 & 130 & 150 & 170 \\
\hline$\alpha_{2}$ & 1 & 1 & 1 & 1 & 1 & 1 & 1 & 1 & 1 \\
\hline$\sigma^{*}$ & 0 & 0 & 0 & 0.03 & 0.06 & 0.08 & 0.10 & 0.11 & 0.12 \\
\hline
\end{tabular}

the teams' abilities. The more asymmetric the teams are, the more likely is it that the FIA changes the rules of the previous season and the more comprehensive are the rule changes. The intuition is also exactly the same as in case of ability regulation. In a rather symmetric contest, competition is already close and the FIA can gain only less with a reduction in discriminatory power of the contest. In contrast, if there are quite asymmetric teams, then the increase in the FIA's revenue due to the improvement of competitive balance is large enough to outweigh the (possible) revenue decrease due to a decline in the performance of the teams.

\section{Empirical Analysis}

In order to empirically test the theoretical model, we offer the following interpretation of the results. Table 1 and 2 show that rule changes are the more likely and the more comprehensive, the larger is the difference in abilities of the racing teams. Moreover, we argued that the difference in abilities is influenced, among other things, by the 
different success of the teams in the previous season. Hence, if competitive balance in the previous season was low (i.e. the difference in success was large), then the difference in abilities in the present season would be large and the FIA is strongly inclined to change rules before the season. In sum, we obtain

Hypothesis $\mathbf{1}$. The smaller competitive balance in season $t-1$, the more likely and the more comprehensive are rule changes at the beginning of season $t$.

A second prediction of the model concerns the impact of rule changes on the equilibrium of the team contest. Equation (16) and (20) show that competitive balance is improved by rule changes and that this improvement is the larger, the more comprehensive the rule changes are. Hence, we obtain

Hypothesis 2. The more comprehensive rule changes are at the beginning of season $t$, the better is competitive balance between the teams during season $t$.

After a rule change the teams will try to improve their performance within the new set of rules. Since a more able team will do this with more success than a less able team, after a while (say, one or two seasons later) competitive balance is worsened again. If the decrease in competitive balance is too large, we return to Hypothesis 1 and the FIA receives an incentive to change rules again. By Hypothesis 2 this would again improve competitive balance and so on. In sum, we obtain a kind of cat-andmouse game between the teams and the FIA. This game might explain the frequent rule changes we observe in the $\mathrm{F} 1$ series.

To test these hypotheses, we use data on F1 seasons from 1950-2003. The data comprise information on safety and technical regulations at the beginning of each season, on points scored by the teams during each season and on the number of fatal accidents during each season. ${ }^{5}$

\footnotetext{
${ }^{5}$ The data on points have been collected from the F1 website at http://www.formulaone.freeonline.co.uk/index.html. The remaining data can be found on the F1Technical website at http://www.f1technical.net. We do not estimate the impact of rules changes on the FIA's broadcasting revenue or the fan interest measured e.g. by the number of viewers. The reason is that the FIA does not publish such data. Some broadcasting stations published data on the number of viewers. But these data are available for a few years and countries only.
} 


\section{$4.1 \quad$ Testing Hypothesis 1}

Hypothesis 1 identifies competitive balance in season $t-1$ as a motivation for rule changes at the beginning of season $t$. The safety of drivers is another motivation for rule changes. We did not take into account this motivation in our theoretical model, but in the empirical analysis we have to control for it since the FIA often justifies rule changes by safety consideration. Hence, the explanatory variables in testing Hypothesis 1 are competitive balance and the safety of drivers in season $t-1$. Competitive balance in season $t-1$ is measured by the standard deviation of points scored by the teams in season $t-1$. This is the same indicator of competitive balance as we used in the theoretical model. ${ }^{6}$ The drivers' safety in season $t-1$ is measured by the number of serious or fatal accidents in season $t-1$.

The dependent variable in testing Hypothesis 1 is the magnitude of rules changes at the beginning of season $t$. Rule changes comprise both the introduction of new rules and the modification or abolition of existing rules. Of course, the rule changes differ with respect to their impact on the outcome of the races. For example, the restriction of the engine capacity usually has another impact as the definition of a maximum bodywork height. But it is impossible to exactly determine the impact of every single rule change. We therefore choose a pragmatic procedure and measure the magnitude of rules changes in season $t$ simply by the number of rules which are modified, introduced or abolished at the beginning of season $t$. The underlying assumption is that the average impact of the rule changes is the same in every season.

The number of rule changes is a discrete variable. Such count data are usually treated within the framework of the Poisson regression model (e.g. Amemiya, 1986, Greene, 1993). Following this approach, we denote the number of rule changes at the beginning of season $t$ by $\mathrm{RC}_{t}$ and the probability that $\mathrm{RC}_{t}$ is equal to $n_{t} \in\{0,1,2, \ldots\}$

\footnotetext{
${ }^{6}$ In the two-player contest, the standard deviation of $p_{i}^{*}$ equals $b^{*} / 2$. The standard deviation of winning percentage is a widely used measure of competitive balance in sports contests (see Fort and Quirk, 1995, and Szymanski, 2003). Other measures include the standard deviation of winning percentage relative to an idealized standard deviation (Scully, 1989, Quirk and Fort, 1992, and Vrooman, 1995), the Gini coefficient (Quirk and Fort, 1992), relative entropy (Horowitz, 1997), the HirschmanHerfindahl index (Depken, 2002) and the ratio of the sum of standard deviations of team performance through time to the sum of within season standard deviations of win percentage (Humphreys, 2002).
} 
by

$$
\operatorname{Prob}\left(\mathrm{RC}_{t}=n_{t}\right)=\frac{e^{-\lambda_{t}} \lambda_{t}^{n_{t}}}{n_{t} !}
$$

where $\ln \lambda_{t}=\boldsymbol{\beta} \boldsymbol{x}_{\boldsymbol{t}-\mathbf{1}}^{\prime}$. The vector $\boldsymbol{\beta}=\left(\beta_{0}, \beta_{1}, \beta_{2}, \beta_{3}\right)$ contains the coefficients to be estimated. The explanatory variables are listed in $\boldsymbol{x}_{t-1}=\left(1, \mathrm{AC}_{t-1}, \mathrm{D}_{t-1} \mathrm{AC}_{t-1}, \mathrm{SD}_{t-1}\right)$ where $\mathrm{SD}_{t-1}$ and $\mathrm{AC}_{t-1}$ denote the standard deviation of points scored by the teams in season $t-1$ and the number of serious or fatal accidents in season $t-1$, respectively. The number of accidents enters our regression directly and by the composed variable $\mathrm{D}_{t-1} \mathrm{AC}_{t-1}$ where $\mathrm{D}_{t-1}$ is a dummy variable equal to 0 for $t \leq 1970$ and 1 for $t>1970$. For the seasons up to 1969, the impact of the number of accidents on the rule setting of the FIA is therefore represented by $\beta_{1}$ while for the seasons after 1969 it is represented by $\beta_{1}+\beta_{2}$. The motivation for this specification is that in the F1 seasons up to 1969 many drivers were killed in the races, but the FIA did not react with safety regulations. Only thereafter the FIA became aware of the danger for the drivers and introduced more safety regulations.

For the interpretation of the coefficients $\boldsymbol{\beta}$, note that $\mathrm{E}\left(\mathrm{RC}_{t}\right)=\operatorname{VAR}\left(\mathrm{RC}_{t}\right)=\lambda_{t}=$ $e^{\boldsymbol{\beta} \boldsymbol{x}_{t-1}^{\prime}}$. It follows $\boldsymbol{\beta}=\left[\partial \mathrm{E}\left(\mathrm{RC}_{t}\right) / \partial \boldsymbol{x}_{\boldsymbol{t}-\mathbf{1}}\right] / \mathrm{E}\left(\mathrm{RC}_{t}\right)$. Hence, $\boldsymbol{\beta}$ shows the percentage change in the expected number of rule changes at the beginning of season $t$ caused by a unit increase in the explanatory variables. Estimating the coefficient yields the results depicted in Table 3. All coefficients are significant and the signs are as expected.

Table 3: Poisson Model for Testing Hypothesis 1

Dependent variable: $\mathrm{RC}_{t}$

\begin{tabular}{lrrrrc}
\hline \hline & coeff. & std.err. & t-statistic & p-value & $95 \%$ conf. interval \\
\hline constant $\left(\beta_{0}\right)$ & 0.444 & 0.092 & 4.800 & 0.000 & {$[0.262 ; 0.625]$} \\
$\mathrm{AC}_{t-1}\left(\beta_{1}\right)$ & -0.211 & 0.026 & -8.21 & 0.000 & {$[-0.261 ;-0.161]$} \\
$\mathrm{D}_{t-1} \mathrm{AC}_{t-1}\left(\beta_{2}\right)$ & 0.431 & 0.026 & 16.360 & 0.000 & {$[0.379 ; 0.483]$} \\
$\mathrm{SD}_{t-1}\left(\beta_{3}\right)$ & 0.028 & 0.002 & 15.270 & 0.000 & {$[0.025 ; 0.032]$} \\
\hline
\end{tabular}

Notes: (i) observations: 53, (ii) log likelihood $=-1592.094$, (iii) pseudo $R^{2}=0.159$.

Before 1970, the lagged number of accidents had a negative impact on the expected number of rule changes, since the FIA did not care about the safety of drivers. The 
impact of the number of accidents on the FIA's rule setting after 1970 is represented by $\beta_{1}+\beta_{2}=-0.211+0.431=0.22$. Hence, an additional fatal accident increases the expected number of rule changes by $22 \%$. This confirms that safety considerations play a role in the rule setting of the FIA. However, the coefficient of the lagged standard deviation of points is also positive. This implies that the expected number of rule changes at the beginning of a season is the larger, the smaller competitive balance has been in the previous season. More specific, a unit increase in the standard deviation of points increases the expected number of rule changes by $2.8 \%$. This result provides empirical evidence for Hypothesis 1 derived from our theoretical model. ${ }^{7}$

The results in Table 3 identify the drivers' safety and competitive balance as motivation for rule changes in the F1 series. To figure out which of these two motivations was more important in the past, we have to compare the number of accidents and the variation in competitive balance. During the seasons 1970-1989, 24 drivers lost their lives in fatal accidents, whereas the standard deviation in the dispersion of points amounted to 8.223. Hence, during this time period the drivers' safety was the main motivation for the frequent rule changes in the F1 series. In contrast, in the seasons 1990-2003 only two drivers were killed, but the standard deviation in the dispersion of points was still 8.211. Consequently, relatively to the drivers' safety, competitive balance became much more important as motivation for rule changes.

\subsection{Testing Hypothesis 2}

The second hypothesis states that rule changes at the beginning of a F1 season improve competitive balance during the season. This hypothesis is tested by an OLS regression model. The number of rule changes at the beginning of a season is chosen as explanatory variable. Since the rule changes in our data set are divided into rule changes regarding the drivers' safety and other rule changes, we use this information and distinguish between the number of safety regulations in season $t, \mathrm{SRC}_{t}$, and the number of other regulations at the beginning of season $t, \mathrm{ORC}_{t}{ }^{8}$ The dependent variable is competitive

\footnotetext{
${ }^{7}$ We also test for overdispersion in the Poisson regression model. The hypothesis that $\mathrm{E}\left(\mathrm{RC}_{t}\right)=$ $\operatorname{Var}\left(\mathrm{RC}_{t}\right)$ cannot be rejected on the basis of the likelihood ratio test.

${ }^{8}$ We did not use this information in testing Hypothesis 1 . The task there was to find out the motivation behind rule changes and distinguishing between types of regulation would bias the results.
} 
balance in season $t$, again measured by the standard deviation of points scored by the teams, $\mathrm{SD}_{t}$. In sum, we obtain the regression equation

$$
\ln \mathrm{SD}_{t}=\beta_{0}+\beta_{1} \ln \mathrm{SRC}_{t}+\beta_{2} \ln \mathrm{ORC}_{t}+\epsilon_{t}
$$

Since this equation is formulated in log-values, the coefficients $\beta_{1}$ and $\beta_{2}$ represent the elasticity of the standard deviation of points with respect to the number of safety regulations and the number of other regulations, respectively.

The results of our estimation are displayed in Table 4 . These results provide clear

Table 4: OLS Model for Testing Hypothesis 2

Dependent variable: $\ln \mathrm{SD}_{t}$

\begin{tabular}{lrrrrc}
\hline \hline & coeff. & std.err. & t-statistic & p-value & $95 \%$ conf. interval \\
\hline constant $\left(\beta_{0}\right)$ & 4.731 & 0.460 & 10.29 & 0.000 & {$[3.691 ; 5.771]$} \\
$\ln \operatorname{SRC}_{t}\left(\beta_{1}\right)$ & -0.263 & 0.150 & -1.76 & 0.112 & {$[-0.602 ; 0.075]$} \\
$\ln \mathrm{ORC}_{t}\left(\beta_{2}\right)$ & -0.524 & 0.241 & -2.18 & 0.058 & {$[-1.070 ; 0.021]$} \\
\hline
\end{tabular}

Notes: (i) observations: 131 ; (ii) $R^{2}=0.3537$

evidence for Hypothesis 2. An increase in the number of rule changes at the beginning of a season makes competition between the F1 teams closer and improves competitive balance during the season. More specific, a $10 \%$ increase in the number of other regulations reduces the dispersion of points by $5.24 \%$. This effect is highly significant at the $5 \%$ level. A little bit surprisingly, even safety regulations have a positive impact on competitive balance. A $10 \%$ increase in the number of safety regulations reduces the standard deviation of points by $2.63 \%$. Although this effect is significant at the $10 \%$ level only, it yet supports Hypothesis 2 and, in addition, may be viewed as a further indication that the motivation behind rule changes is not always the safety of drivers: Even if a rule change is declared as safety regulation, it helps to render the F1 championship closer and, thus, the true motivation behind the rule change might be competitive balance between the teams instead of the drivers' safety. 


\section{Conclusion}

This study provides for the first time an economic analysis of the F1 motor racing series. The main question was to theoretically and empirically determine the motivation for the frequent rule changes in the F1 series. In the first part, we developed a theoretical model in which the motivation for rule changes was competitive balance between the teams. In this model, a rule change at the beginning of a F1 season has two effects. It reduces the performance of the racing teams and improves competitive balance. The broadcasting revenue of the organizer, the FIA, is reduced by the former effect and increased by the latter. Hence, if the revenue gain from the increase in competitive balance is sufficiently large, the FIA receives an incentive to change the rules. In the second part, we empirically estimated this theory for the F1 seasons 1950-2003. It turned out that, beside the drivers' safety, competitive balance is a significant determinant of the rule setting behavior of the FIA. The expected number of rule changes at the beginning of a season is high, when competitive balance in the previous season has been low. It was also shown that rule changes at the beginning of a season exert a significant positive impact on competitive balance during the season, even if the rule changes are declared as safety regulation.

What is the current situation in the F1 series? During 1999-2002 there was a dominance of the Ferrari team which won the constructors' championship and also the drivers' championship (with the exception of 1999). Consistent with our analysis, the FIA implemented comprehensive rule changes at the beginning of the season 2003 and, even though Ferrari won the titles also in 2003, the championship in this season was much more balanced. But in the current season (2004) the Ferrari team regains its dominance. Its driver Michael Schumacher already won 8 out of the first 9 races. If this development continues, then we expect comprehensive rule changes in one of the next seasons and perhaps they will look like those proposed by Bob Kravitz, the commentator of The Greenville News: 'Next year Michael Schumacher must drive with a bag of groceries at his knees and a cell phone affixed to his ear. Or maybe, just to make it interesting, he should be forced to drive in the other direction.' 


\section{References}

Amegashie, A. (1999), The Design of Rent-Seeking Competitions: Committees, Preliminary and Final Contests, Public Choice 99, 63-76.

Amemiya, T. (1986), Advanced Econometrics, Basil Blackwell.

Dasgupta, A., and K.O. Nti (1998), Designing an Optimal Contest, European Journal of Political Economy 14, 587-603.

Depken, C. A. (2002), Free Agency and the Concentration of Player Talent in Major League Baseball, Journal of Sports Economics 3, 335-353.

FIA (1999), The Economic Impact of European Grand Prix, Technical report, Paris. Fort, R. and J. Quirk (1995), Cross Subsidization, Incentives and Outcomes in Professional Team Sports Leagues, Journal of Economic Literature 33, 1265-1299.

Greene, W.H. (1993), Econometric Analysis, Second Edition, MacMillan.

Humphreys, B.R. (2002), Alternative Measures of Competitive Balance in Sports Leagues, Journal of Sports Economics 3, 133-148.

Horowitz, I. (1997), The Increasing Competitive Balance in Major League Baseball, Review of Industrial Organization 12, 373-387.

Michaels, R. (1988), The Design of Rent-Seeking Competitions, Public Choice 56, $17-29$.

Quirk, J., and R. Fort (1992), Pay Dirt: The Business of Professional Team Sports, Princeton University Press.

Runkel, M. (2003), Optimal Contest Design When the Designer's Payoff Depends on Competitive Balance, CESifo Working Paper No. 1009, Munich.

Scully (1989), The Business of Major League Baseball, University of Chicago Press.

Szymanski, S. (2003), The Economic Design of Sporting Contests: A Review, Journal of Economic Literature 41, 1137-1187. 
Tullock, G. (1980), Efficient Rent-Seeking, in: Buchanan, J.M., Tollison, R.D., and G. Tullock (eds.), Toward a Theory of the Rent-Seeking Society, College Station: Texas A\&M University Press.

Vrooman, J. (1995), A General Theory of Professional Sports Leagues, Southern Economic Journal 61, 971-990. 